\section{From Bone Cell Biology to Novel Therapies of Osteoporosis}

\author{
L. C. Hofbauer ${ }^{1}$ \\ ${ }^{1}$ Medizinische Klinik u. Poliklinik III, Universitätsklinikum \\ Carl Gustav Carus, Technische Universität Dresden
}

Osteoporosis is a common disease characterised by a systemic impairment of bone mass and microarchitecture that results in fragility fractures. With an ageing population, the medical impact of osteoporosis will increase further. A detailed knowledge of bone biology with molecular insights into the communication between bone-forming osteoblasts and bone-resorbing osteoclasts and the orchestrating signalling network has led to the identification of novel therapeutic targets. Based on this, novel treatment strategies have been developed aimed at inhibiting excessive bone resorption and increasing bone formation. The most promising novel treatments include denosumab, a monoclonal antibody against receptor activator of NF-KB ligand, a key osteoclast cytokine, odanacatib, a specific inhibitor of the osteoclast protease cathepsin $\mathrm{K}$, and antibodies against the sclerostin, an endogenous inhibitor of bone formation.

In the past decade, the pathogenesis of osteoporosis has been linked to processes at the tissue, cellular, and molecular levels. Signals that act as „master-switches“ have been defined that integrate various endocrine, neuroendocrine, inflammatory, and mechanical stimuli. At the cellular level, communication and coupling between the main bone cell types, the bone-forming osteoblasts and the bone-degrading osteoclasts constitute the smallest functional unit. Several key molecules conduct the coordinated activities of osteoblasts and osteoclasts during bone remodeling. Detailed knowledge of the molecular and cellular players has created a new concept of bone pathophysiology. With some of these new principles finding their way into clinical practice, we will highlight the most relevant advances in the field.

Osteoclasts originate from haematopoietic stem cells and are closely related to monocytes and macrophages. Differentiation from osteoclast precursor cells to fully activated multinucleated osteoclasts depends critically on the presence of receptor activator of NF-KB ligand (RANKL), a member of the TNF family, and the permissive role of macrophage colony-stimulating factor (M-CSF). Cathepsin K represents a key cystein proteinase of the mature osteoclast that degrades collagen and breaks down bone. Cathepsin $\mathrm{K}$ is a critical determinant of resorptive activity by osteoclasts which removes bone of poor quality, where micro-cracks have accumulated and leaves hole-like lacunae. The osteoblast represents a unique bone-forming cell derived from mesenchymal stem cells. The speed and efficacy of precursor cells differentiating into mature osteoblasts that secrete matrix that can be mineralised and their life span determines the rate of bone formation. At the molecular level, activation of the canonical Wnt/ $\beta$-catenin pathway is the master switch for osteoblastic differentiation. This key bone-anabolic pathway is negatively regulated by Wnt inhibitors such as dickkopf-1
(Dkk-1) and sclerostin which bind and block the Wnt receptor LRP-5.

\section{Denosumab}

The prominent role of RANKL in osteoclastogenesis has made it a prime target for therapy against diseases characterised by excessive bone loss. This led to the development of denosumab, a fully human monoclonal antibody against RANKL. Denosumab displays a high specificity and affinity for RANKL, translating into a longer dosing interval of 6 month. A large study program on a wide spectrum of bone diseases, including several types of osteoporosis and bone metastases is currently ongoing. With completed phase 3 studies, denosumab is the most advanced of all investigational substances and has 2010 been approved in Europe and the US for the treatment of osteoporosis.

In the phase 1 study, a single subcutaneous injection dosedependently suppressed urinary N-terminal telopeptide of type I collagen (NTX), a biochemical marker of bone resorption, by up to $81 \%$ in postmenopausal women for as long as 6 months and was well-tolerated. Subsequently, a phase 2 study with different doses (6-210 mg) and intervals (every 3 to 6 months) was aimed at evaluating the effects on BMD after 12 months in postmenopausal women with low bone mass. A dose-dependent suppression of bone turnover was observed, with a decrease of the bone resorption marker serum CTX as early as three days after administration and a maximum reduction of $88 \%$ in the denosumab groups. Of note, discontinuation of denosumab treatment caused a rapid increase of bone turnover markers to values above baseline and even greater than those observed in the placebo group. Long term follow-up is required to determine the clinical effects of suppressed bone turnover during denosumab therapy.

In a pivotal randomised placebo-controlled phase 3 study (FREEDOM), denosumab was assessed for its fracture reduction in 7,868 women with postmenopausal osteoporosis, of whom $24 \%$ had preexisting vertebral fractures. After 3 years, denosumab had reduced the risk of new radiographic vertebral fractures by $68 \%$, hip fractures by $40 \%$, and non-vertebral fractures by $20 \%$. Of note, the risk of cardiovascular events, cancer, and infections did not differ between the two groups. Comprehensive assessment of the immune status of patients receiving denosumab for 12 months revealed no relevant changes in blood cell count. In the follow-up of the FREEDOM cohort, one woman developed osteonecrosis of the jaw after dental extraction. Osteonecrosis of the jaw has been previously described as a rare complication with a frequency of $1: 100,000$ to $1: 10,000$ in patients treated for osteoporosis with bisphosphonates.

In summary, denosumab represents a novel and effective anti-resorptive therapy for various metabolic bone diseases. While direct comparative studies with fracture endpoints are not available, evidence from completed trials with established surrogates suggests that it may be as effective as the most potent of the amino-bisphosphonates, zoledronic acid. Denosumab can be considered as a first line therapy. In addition, it can be given as an alternative to intravenous bisphosphonates or if oral bisphosphonates are not tolerated. 


\section{Odanacatib}

Based on the concept that the protease cathepsin K plays an important role in enzymatic bone degradation, the use of cathepsin $\mathrm{K}$ inhibitors has emerged as a novel therapeutic approach. Odanacatib is currently the most advanced cathepsin $\mathrm{K}$ inhibitor under clinical investigation. Programs with less specific cathepsin $\mathrm{K}$ inhibitors were stopped due to cutaneous adverse side effects. In phase 1 studies, odanacatib at an oral dose of 50 and $100 \mathrm{mg}$ once a week reduced serum levels of the bone resorption marker C-terminal telopeptide of type I collagen (CTX) by $62 \%$. Daily administration of odanacatib $(10 \mathrm{mg})$ reduced serum CTX by $81 \%$. In a phase 2 study, the effects of weekly oral doses of odanacatib were assessed in 399 women with postmenopausal osteoporosis. After 24 months, odanacatib (50 mg) increased the BMD of the lumbar spine and total hip by $5.7 \%$ and $4.1 \%$ compared to placebo, respectively. Bone resorption markers were dose-dependently suppressed. Of note, odanacatib treatment resulted in a modest and transient reduction of bone formation markers while not suppressing bone formation. Adverse reactions were comparable to placebo and scleroderma-like cutaneous lesions were not observed. Currently, a phase 3 study is being conducted with over 16,000 postmenopausal women to assess the anti-fracture efficacy of odanacatib. Another cathepsin K inhibitor, ONO-5334 is currently evaluated in a phase 2 trial in women with postmenopausal osteoporosis (NCT 00532337).

\section{Sclerostin antibody}

Wnt-dependent nuclear accumulation of $\beta$-catenin is a major trigger of osteoblastic differentiation and bone formation. The endogenous inhibitors of Wnt signalling, sclerostin and Dkk-1 present potential therapeutic targets to enhance osteoblastic bone formation and are under clinical investigation. Two rare skeletal diseases with a high bone mass, van Buchem disease and sclerosteosis, have been linked to inactivating mutations in the gene encoding for sclerostin. This highlights the role of sclerostin in the homeostasis of bone mass, and provided the rationale to target sclerostin with monoclonal antibodies to enhance bone formation. In a rat model of postmenopausal osteoporosis due to ovariectomy, treatment with a sclerostin antibody increased bone mass at all skeletal sites and completely prevented bone loss associated with estrogen deficiency. Treatment of cynomolgus monkeys with two once-monthly injections of a sclerostinneutralising antibody yielded an increased bone mass at the femoral neck, radius, and tibia, and enhanced bone strength at the lumbar spine. In a phase 1 study, a single subcutaneous injection of a sclerostin antibody was well tolerated and increased bone formation. Of note, the combination of stimulated bone formation and unchanged bone resorption markers indicates an uncoupling effect. A phase 2 trial has been initiated to compare the efficacy of sclerostin neutralisation with alendronate and teriparatide.

Conflict of Interest: Professor Hofbauer has received honoraria for lectures and advisory boards for Amgen, Merck, Novartis and UCB as well as research support from Amgen and Novartis to his institution.

\section{Bibliography}

Dol http://dx.doi.org/10.1055/s-0035-1558064

Drug Res 2015; 65, Suppl. 1: S14-S15

(c) Georg Thieme Verlag KG Stuttgart · New York .

ISSN 2194-9379

Correspondence

Prof. Dr. Lorenz C. Hofbauer

Medizinische Klinik u. Poliklinik III

Universitätsklinikum Carl Gustav Carus

Technische Universität Dresden

Fetscherstraße 74

01307 Dresden

lorenz.hofbauer@uniklinikum-dresden.de

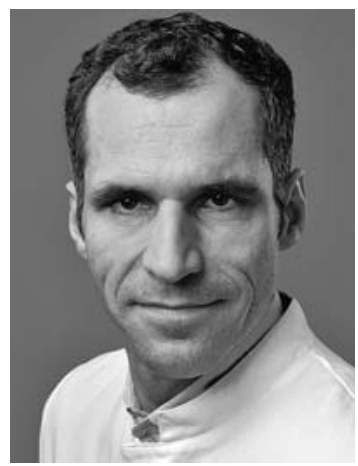

